\title{
Modeling and Generating Organ Pipes Self-Sustained Tones by Using ICA
}

\author{
Angelo Ciaramella ${ }^{1}$, Enza De Lauro ${ }^{2}$, Salvatore De Martino², Mariarosaria Falanga ${ }^{2}$, \\ Roberto Tagliaferri ${ }^{3}$
}

${ }^{1}$ Department of Applied Science, University of Naples “Parthenope,” Centro Direzionale, Naples, Italy; ${ }^{2}$ University of Salerno, Fisciano, Italy; ${ }^{3}$ Department of Computer Science, University of Salerno, Fisciano, Italy.

Email: angelo.ciaramella@uniparthenope.it, \{edelauro, sdemartino,mafalanga,rtagliaferri \}@unisa.it

Received June $7^{\text {th }}, 2011$; revised July $10^{\text {th }}, 2011$; accepted July $19^{\text {th }}, 2011$.

\begin{abstract}
Aim of this work is to analyze and to synthesize acoustic signals emitted by organ pipes. An Independent Component Analysis technique is applied to study the behavior of single notes or chords obtained in real and simulated environments. These analyses suggest that the pipe acoustic signals can be described by a mixture of nonlinear oscillations obtained by a self-sustained feedback system (i.e., Andronov oscillator). This system allows to obtain a realistic pipe waveform with features very similar to the sound produced by the pipe and to propose an additive synthesis model. Moreover, suitable analogical and integrate circuit models, able to reproduce the registered waveforms and sound, have been designed. A comparison between real and reconstructed acoustic signals is provided.
\end{abstract}

Keywords: Independent Component Analysis, Sound Synthesis, Non-Linear Oscillators, Integrate Circuits

\section{Introduction}

One of the challenge of the researchers working in music field is to generate and to control the sound. A synthesizer (or synthesiser) is an electronic instrument able to reproduce musical sounds. Sound can be produced by electrical oscillators which are fed to filters (analog synthesizer), or by performing mathematical operations in a microprocessor (digital synthesizers). Both analog and digital synthesized sounds may sound dramatically different than recordings of natural sounds. There are also many different kinds of synthesis methods, each applicable to both analog and digital synthesizers. These techniques tend to be mathematically related, especially the frequency modulation and the phase modulation. Examples of these methods are subtractive, frequency modulation, physical modeling, sample based synthesis and so on [1]. We also note that the sound envelope is used in many synthesizers, samplers, and other electronic musical instruments. Its function is to modulate some aspects of the instrument's sound. The envelope may be a discrete circuit or module (in the case of analog devices), or implemented as part of the unit software (in the case of digital devices). When a mechanical musical instrument produces sound, the volume of the sound produced changes over time in a way that varies from instrument to instrument. The envelope is a way to tailor the timbre for the synthesizer, sometimes to make it sound more like a mechanical instrument. For example, a quick attack with little decay helps it to play more like an organ; a longer decay and zero sustain makes it more like a guitar. In any case, any method to synthesize sound is based on a physical model. The first modeling of the sound production and of the acoustics of the musical instruments is the linear harmonic approximation. This approximation can be suitable for some instruments such as guitar, piano, etc., whereas it fails for other instruments such as wind instruments, whose sound is produced by a nonlinear mechanism [2,3].

In this paper, we study the organ pipes that are particular wind instruments in which the nonlinearity does not depend on the coupling between instrument and player but it is intrinsic. The now working model of the organ pipes relies upon the mode-mode coupling and multiphonic sound production [4]. We investigate the real acoustic signals emitted by organ pipes with the aim to reproduce their emitted sound. We propose an additive synthesis model based on a simple valve analogical circuit. This model is able to reproduce sounds similar to the recoded ones both in the waveforms and in the listening. To reach this goal, we have recorded signals in several experiments and we have applied well established 
techniques of nonlinear processing operating in the time domain. A particular role in this analysis is covered by the Independent Component Analysis (ICA) [5]. It allows to establish whether the experimental time series are a linear superposition of statistically independent sources. The other step is the noise reduction of the decomposed signals [6]. Indeed, in experimental data, even decomposed into simpler sources, a contribution of residual noise is still relevant.

The de-noised components are the basis for the reconstruction of the phase space [7-9]. The estimation of the embedding dimension and of the other extracted information allow to recognize the class of dynamical systems that characterizes the dynamics generating the experimental series [10].

Finally, numerical simulations and comparative simple methods lead to very simple equations that reproduce signals even in the listening as confirmed by a formal AB-preference test [11]. These equations, i.e., a low dimension dynamical system, represent on average the behavior of the complete fluid-dynamical equations describing the phenomenon. We stress that this model provides a nonlinear waveform that is highly correlated with the original pipe sound signal (including the envelope). In other words, we can reproduce the timbre of the pipe sound including the attack, decay, sustain and release phases by using an analogical circuit.

The paper is organized as follows. In Section 2 we insert a brief introduction of the organ pipes. In Section 3 we focus our attention on the description of the wind instruments remarking their nonlinear features. In Section 4 we introduce the ICA approach and the FastICA algorithm, and in Section 5 a noise reduction method is described. In Section 6 we present the experiments obtained on real data and we propose the analogical model to reproduce the registered waveforms. The conclusions are described in Section 8.

\section{Organ Pipe Ranks}

The pipe organ is essentially a mechanized wind instrument of the panpipe type. Each pipe is a simple sound generator optimized to produce just one note with a particular loudness and timbre, and the organ mechanism directs air to particular combinations of pipes to produce the desired sound. A set of pipes of uniform tone quality, with one pipe for each note over the compass of the organ keyboard, is called rank. The pipes are set out logically, and generally to a large extent physically, in a matrix. Each row of the matrix contains the pipes of a single rank and each column of the matrix contains all the pipes for a single note. There are two types of ranks: flues and reeds. Flue pipes, also called labial (the upper lip of the mouth is important in sound production) belong to the flute-instrument family. Open flue pipes are historically the basis of the pipe organ and still provide its foundation sound. We can also have stopped flue pipes [3]. In addition, there are various partly stopped pipes in which the stopper has a vent or chimney to produce special effects. Reed pipes, or lingual, have a metal tongue vibrating against a rather clarinet-like structure called a shallot. There are two major classes of reeds: those with full length conical resonators supporting all harmonics and those half-length cylindrical resonators supporting primarily the odd harmonics. In addition, we find short reed pipes with cavity resonators rather like trumpet mutes, but they are quite unusual in modern organs. In virtually all natural sounds, increase loudness is associated not simply with a uniform increase in sound pressure level at all frequencies, but rather with a change in the slope of the frequency spectrum to give more weight to components of higher frequencies. This is a natural conesquence of the nonlinearities associated with the production of such sounds. We also note that the air column in a cylindrical pipe is only approximately harmonic in its resonances [12]. In [3] is demonstrated the mechanism that provides considerable nonlinearity for the generation of the harmonics which are then amplified by the resonator (or not amplified, in the case of even harmonics and a stopped cylindrical pipe). It also provides the mechanism for mode locking and, when the conditions are satisfied, for multiphonic production. The pipe organ is a very complex instrument, with thousands of pipes at a multitude of pitches, all under the control of a single organist.

\section{Sustained-Tone Instruments and Nonlinearity}

Musical instruments are often thought as linear harmonic systems. A closer examination, however, shows that the reality is very different from this.

Sustained-tone instruments, such as violins, flutes and trumpets, have resonators that are only approximately harmonic, and their operation and harmonic sound spectrum both rely upon the extreme nonlinearity of their driving mechanisms. It is helpful to consider the whole system made of the sustained-tone instrument and its player, as shown in Figure 1. The instrument itself generally has a primary harmonic resonator that is maintained in oscillation by a power source provided by the player, together with a secondary resonator, generally with some broad and inharmonic spectral properties, that acts as a radiator for the oscillations of the primary resonator. In the linear harmonic approximation, the generator is assumed simply to provide a negative resistance to overcome the mechanical and acoustic losses in the primary resonator, but no information is provided about the 


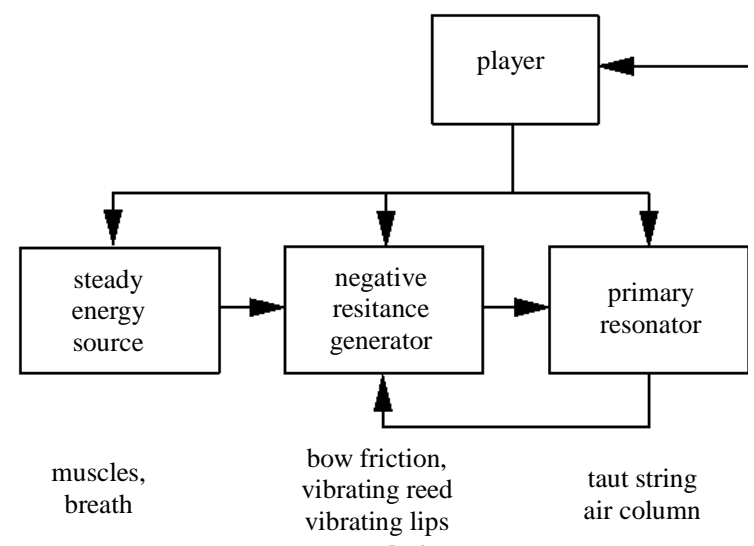

Figure 1. System diagram for a sustained-tone musical instrument. In most cases the generator is highly nonlinear and all the other elements are linear.

spectral envelope, and thus about the tone quality of the sound.

Wind instruments are rather different, in that the primary resonating body is a air column, which also radiates the sound. There is therefore one less element in the total system diagram. In the more detailed nonlinear treatment, the generator is usually highly nonlinear, and it is strongly coupled with the primary resonator. The latter is usually appreciably inharmonic in its modal properties. The feedback coupling between the resonator and the generator therefore assumes prime importance in determining the instrument behavior.

\section{The ICA Method}

ICA is a method to find underlying factors or components from multivariate (multidimensional) statistical data, based on their statistical independence [5]. In the simplest form of ICA, one observes $m$ scalar random variables $x_{1}, x_{2}, \cdots, x_{m}$ which are assumed to be linear combinations of $n$ unknown independent components (ICs) denoted by $s_{1}, s_{2}, \cdots, s_{n}$. These ICs $s_{i}$ are assumed to be mutually statistically independent, and zero-mean. Arranging the observed variables $x_{j}$ into a vector $\boldsymbol{x}=\left(x_{1}, x_{2}, \cdots, x_{m}\right)^{\mathrm{T}}$ and the IC variables $s_{i}$ into a vector $\boldsymbol{s}$, the linear relationship can be expressed as $\boldsymbol{x}=\boldsymbol{A s}$. Here $\boldsymbol{A}$ is an unknown $m \times n$ matrix of full column rank, called the mixing matrix. The basic problem of ICA is then to estimate both the mixing matrix $\boldsymbol{A}$ and the realizations of the ICs $s_{i}$ using only observations of the mixtures $x_{j}$. Estimation of ICA requires the use of higher-order statistical information. Some heuristic approaches have been proposed in literature for achieving separation and some authors have derived "unsupervised neural" learning algorithms from information-theoretic measures. Among them, a good measure of independence is given by negentropy. In the following we shall use the fixed-point algorithm, namely FastICA, developed to perform linear mixtures separation by using the negentropy information [5].

ICA has revealed many interesting applications in different fields of research (bio-medical signals, geophysics, audio signals, image processing, financial data, etc.). For instance, it was fruitfully applied in volcanic environment $[8,13]$, physics of musical instruments $[12,14,15]$ and dynamical systems in mixtures [16]. Moreover, further studies have been conducted on signals recorded in real environments with delay and reverberation (e.g., convolutive mixtures) $[17,18]$.

\section{Noise Reduction}

The method used in the experiments to accomplish the nonlinear noise reduction (RNRPCA in the following) is based on the application of the compression and decompression (reconstruction) of the noise data [6]. To estimate the noise strength we followed the heuristic method of Natarajan [19]. Many runs of compression/decompression algorithms of various compression losses, measured as Peak Signal-to-Noise Ratio (PSNR), have been tried. After all runs one has to plot the compression ratio versus PSNR values to obtain the rate-distortion characteristic of the signal. At the point of PSNR corresponding to the strength of the noise, the plot of the noisy signal shows the knee point, that is a point at which the slope of the curve changes rapidly. The precise determination of the knee point can be obtained by drawing the second derivative. The point of PSNR at which the second derivative attains its maximum is the measure of the noise strength. The practical solution of filtering the random noise has been obtained through the use of Robust Principal Component Analysis Neural Network [14].

\section{Experimental Results}

The aim of the following experiments is to analyze the data recorded playing organ pipes, by using the ICA approach in order to establish how many independent components, if there are, are necessary to retain all the information of the recorded signals.

\subsection{Recording Data}

The first part of our work examines the sound recordings performed in the church of St. Antonio di Padova located in Mercato San Severino (SA). We have used a digital acquisition board with a sampling frequency of 44,100 $\mathrm{Hz}$ and nine microphones. The organ is located at three meters from the principal floor of the church. The microphones have been positioned linearly along the principal floor. Three clusters, composed of three microphones each, have been posed five meters far each other. In this setup, we have recorded nine signals in each ex- 
periment, recording the notes $C, E$, G. Furthermore, we have also performed experiments playing chords. A preliminary analysis of this experimental survey is contained in [10]. The second part of our work looks at the experiments performed playing an organ pipe in the acoustic laboratory of Salerno University, where we have used the same acquisition board and six microphones (see Figure 2 for details of the organ pipes). In these experiments, we have recorded single notes played with three different levels of the blowing pressure in order to evidence how the frequencies depend on the forcing. The pipe blowing pressure has been supplied by a standard organ mechanism in the church, whereas it is induced by the human breath at the Department. At the end of the complete survey, our data set is composed of many scalar series for each note. The notes are produced by using organ pipe in different registers and octaves.

\subsection{Data Analysis}

In the first experiment we focus our attention to analyze the notes and chords obtained by the organ pipe. The aim is to analyze this kind of sources, using the FastICA approach, to study the features of the Dynamical Systems (DSs) that generate these signals. Our data set, taking into account the linear geometry of the microphones, can be viewed as different spatial records of the same physical phenomenon. Before applying FastICA we need to align the recordings, using cross-correlation, to avoid the delays among the microphones. Analyzing the geometry of the sampling we conclude that the delay and the reverthe microphones, the environment and the features of

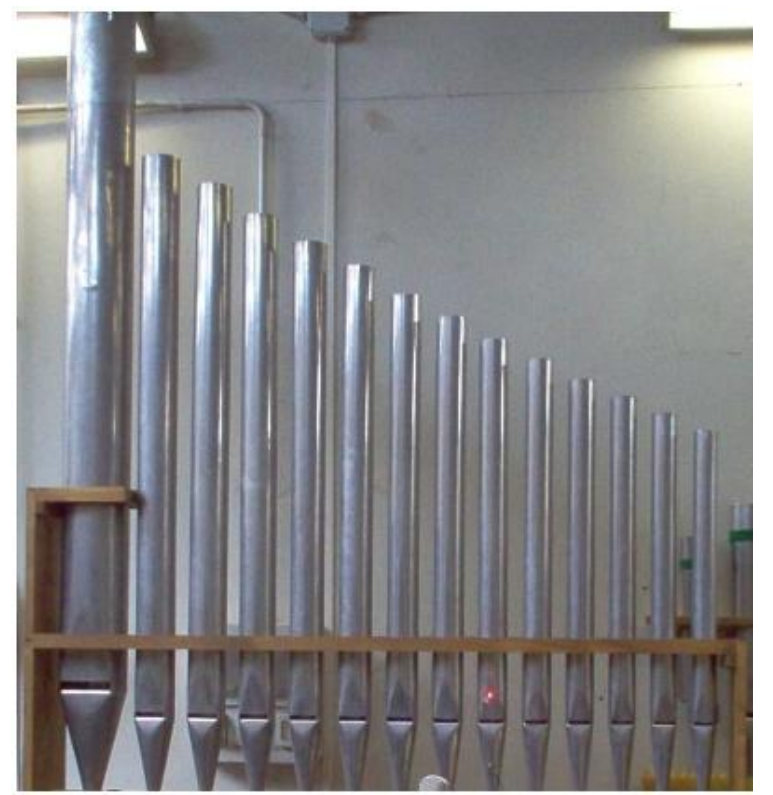

Figure 2. Organ pipes in the acoustic Laboratory of Salerno University. beration in this case can be neglected and then we can consider a generative model based on instantaneous mixtures. In our analysis first of all we focus the attention on the single $C$ note. In Figure 3(a), 9 recorded signals for the $C$ note and the relative Power Spectrum Densities (PSDs) are reported. In this case the fundamental frequency is at $523 \mathrm{~Hz}$. Applying the FastICA approach to the nine recorded signals we obtain the separation of three waveforms with the fundamental frequency at 523 $\mathrm{Hz}$ and other frequency peaks (see Figure 3(b)). They are nonlinear signals in limit cycle regime that are linearly superimposed. The study of principal components of the covariance matrix of the signals reveals that there are only six principal components. Using this information and applying to these signals the noise reduction method RNRPCA, we obtain a more clear separation, as we can see in Figure 3(c). The separated signals are the fundamental of the $C$ note with frequency at $523 \mathrm{~Hz}$, one waveform with lower frequency at $263 \mathrm{~Hz}$ and another at $1046 \mathrm{~Hz}$. Moreover, the FastICA approach has extracted two independent signals more with frequencies of $98 \mathrm{~Hz}$ and $784 \mathrm{~Hz}$. The signal with a main peak equal to $98 \mathrm{~Hz}$ can be ascribed the power supply or to $G$ note, whereas $784 \mathrm{~Hz}$ signal can be a harmonic of $C$ or another $G$ pipe in resonance.

In the second experiment, we have considered chords. In particular, we have played the $C, E$ and $G$ notes simultaneously. The estimated sources are related to the principal modes $(C=523 \mathrm{~Hz}, E=331 \mathrm{~Hz}$ and $G=393$ $\mathrm{Hz}$ ) and other frequency peaks $(C=262 \mathrm{~Hz}, E=663 \mathrm{~Hz}$, $G=784 \mathrm{~Hz}$ and $G=98 \mathrm{~Hz}$ ). Also in this case we have applied the RNRPCA algorithm obtaining clearer results. Coming to the laboratory experiments, we have played organ pipes under controlled conditions looking at the sound produced by a single organ pipe without the presence of the others which could influence the sound. We have recorded many pipes played with three levels of blowing pressure: full-toned, intermediate and low level of pressure. For brevity we report the results relative to the $E$ note stressing that the acoustic field produced by the other pipes displays the same features. The recordings are preprocessed to avoid the contribution of the power supply, high-pass filtering the experimental signals over $50 \mathrm{~Hz}$. To get some knowledge about the dynamics generating acoustic signals we have estimated their phase space and the embedding dimension. The standard techniques to reconstruct the phase space, based on the theorem of Takens [9], are well known [7]. To estimate an upper bound of the attractor dimension, we used the False Nearest Neighbors (FNN) techniques and the Average Mutual Information (AMI) [20].

In the experiments illustrated in this paper the embedding dimension of the notes is 4 . 

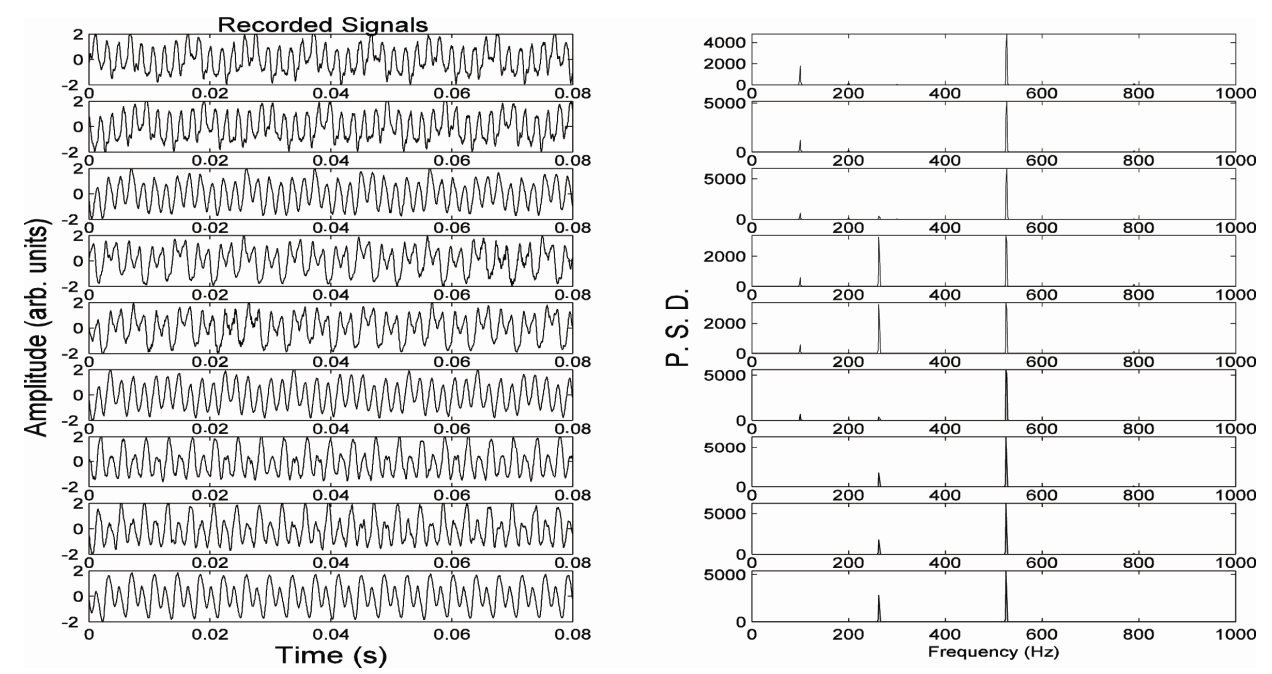

(a)
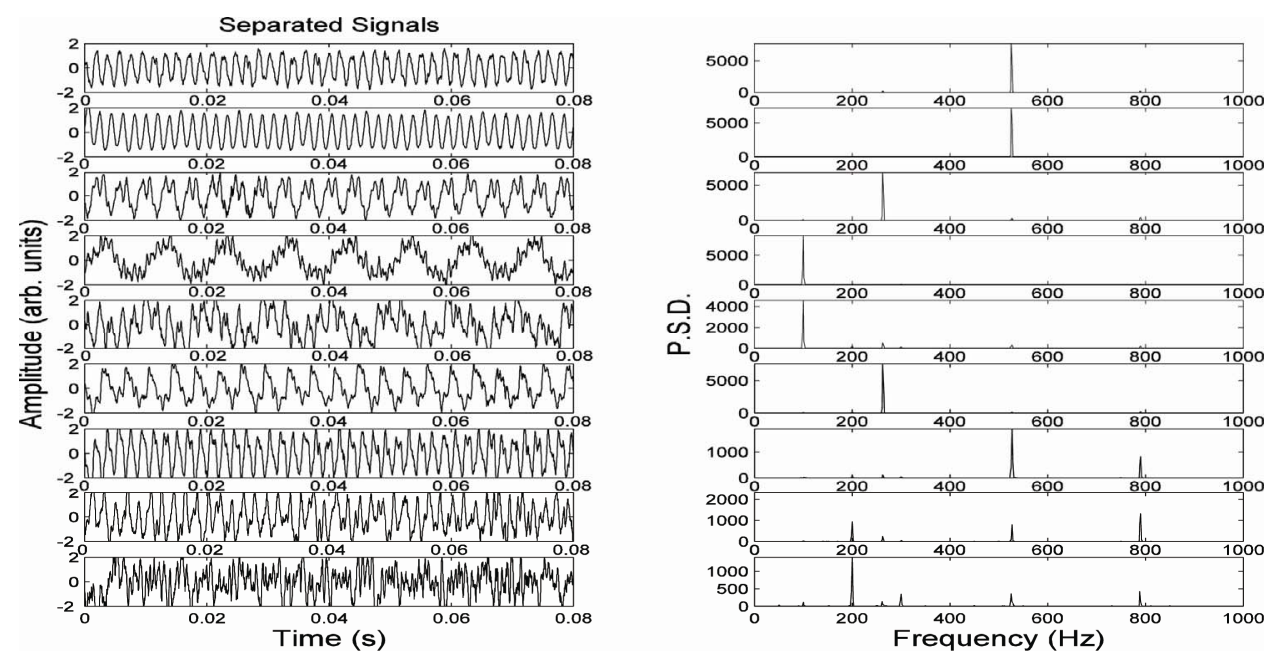

(b)
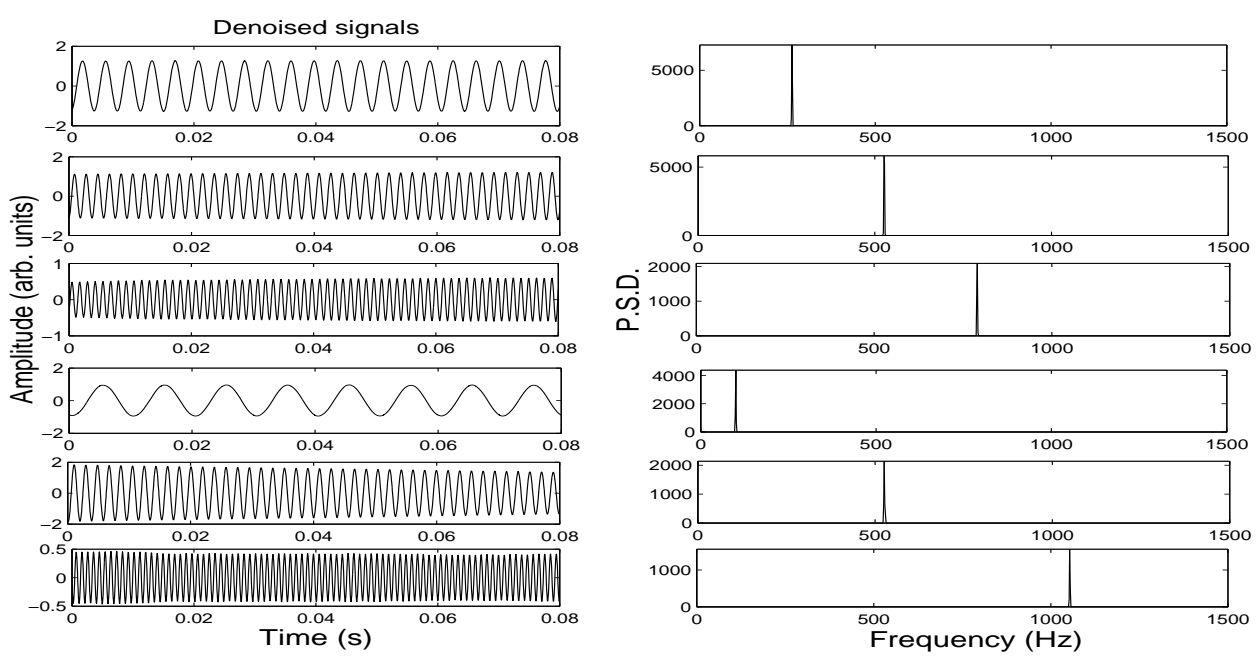

(c)

Figure 3. C note source separation (recorded in the church): (a) recorded signals; (b) estimated signals; (c) signals obtained applying the noise reduction method RNRPCA to the Independent extracted Components. 
In Figures 4(a)-(c), we can see the results of the $E$ note full-toned played. In Figure 4(b) we have the eigenvalues of the PCA. If we look at Figure 4(c), i.e., the ICA extracted components, we recover three modes at the appropriate frequencies (principal mode at $331 \mathrm{~Hz}$, higher at $663 \mathrm{~Hz}$, lower at $165 \mathrm{~Hz}$ ), corresponding to $E$ note. When an isolated organ pipe is played in standard condition there are always present three nonlinear modes [21]. We reduce the blowing pressure at the intermediate value, as one can observe in Figures 5(a)-(c), until to excite just two nonlinear modes: the principal and the higher mode. Finally, at the low level of pressure, just one mode is activated as clearly shown in Figures 6(a)-(c).

These analyses suggest to synthesize the pipe organ sound by using an additive synthesis, taking into account the independent components information of the sound in order to obtain a better sound quality. In other words, to obtain the full toned voice of an organ pipe, we need to excite three modes. Although the signals could appear as composed by a mixture of linear oscillators, by ICA we have proved that they result a mixture of nonlinear oscillators.

Summarizing, ICA establishes that the sound produced by a full-played single organ pipe can be modeled by a low-dimensional dynamical system. Furthermore, this system is the superposition of three nonlinear oscillators, in self-oscillating regime.

\section{Analogical Model and Circuit}

By using ICA we have had information about the dynamic system involved in the generation of sound. On this basis, we propose an analogical model able to reproduce in the listening the recorded notes. This model represents the simplest nonlinear dynamical system, that can generate "harmonicity". This system works with a feedback and produces self-sustained oscillations [22] under suitable parameters (Andronov oscillator). We remind that a limit cycle which is asymptotically approached by all the other phase paths and it is dynamically stable. A simple example of application is the valve oscillator with the oscillating $R L C$ circuit in the anode circuit and an inductive feedback on the grid (see Figure 7). Simple mathematical equations can be obtained by neglecting the anode conductance, the grid currents and the inter electrode capacitances, and assuming a piecewise linear approximation for the valve characteristic $i_{a}=$ $i_{a}(u)$, where $u$ is the grid voltage and $i_{a}$ is the anode current. Under these hypotheses, the equations of the Andronov oscillator are:

$$
\begin{aligned}
& L C \ddot{u}+R C \dot{u}+u=M \frac{\mathrm{d} i_{a}}{\mathrm{~d} t} \\
& i_{a}=f(u)= \begin{cases}0 & u<-u_{0} \\
S\left(u+u_{0}\right) & u>-u_{0}\end{cases}
\end{aligned}
$$

where $M$ is the mutual inductance (which has to be negative to install self-coupling; $S$ is the positive slope of the

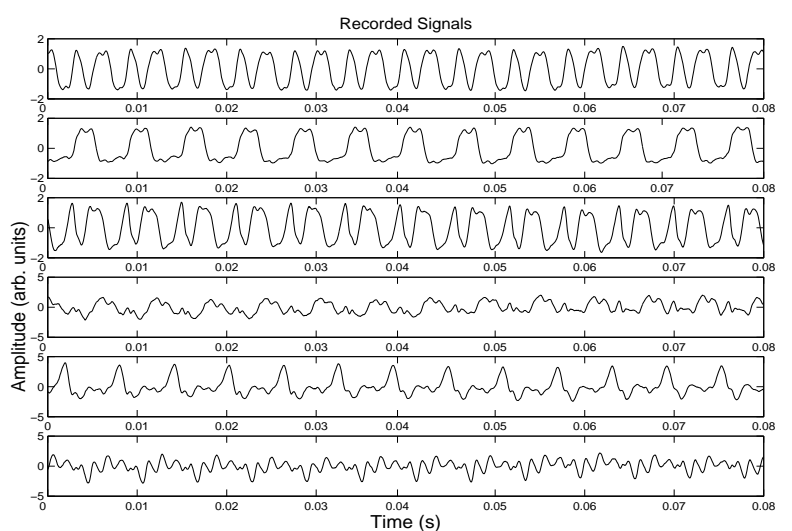

(a)

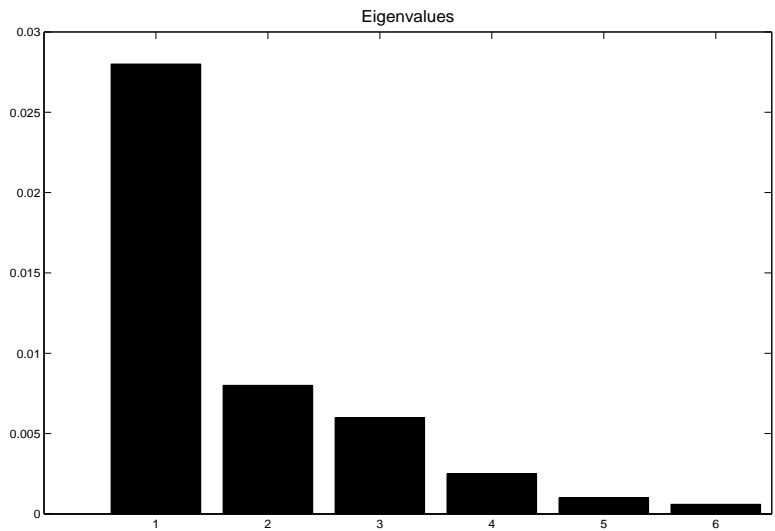

(b)

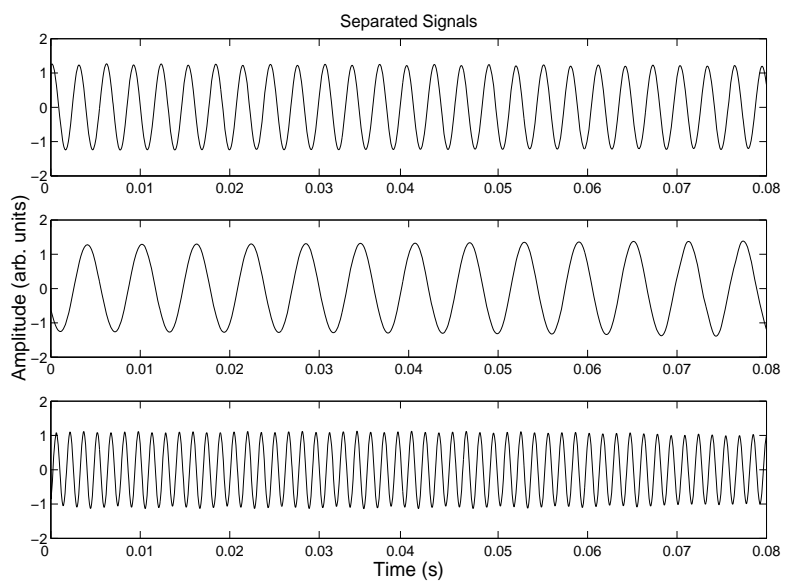

(c)

Figure 4. Separation related to $E$ note produced by a pipe when it is full toned (played in the laboratory): (a) original signals; (b) PCA: eigenvalues of the covariance matrix; (c) signals separated by ICA with principal peak at $331 \mathrm{~Hz}$ and lower and higher modes respectively at $165 \mathrm{~Hz}$ and $663 \mathrm{~Hz}$. 


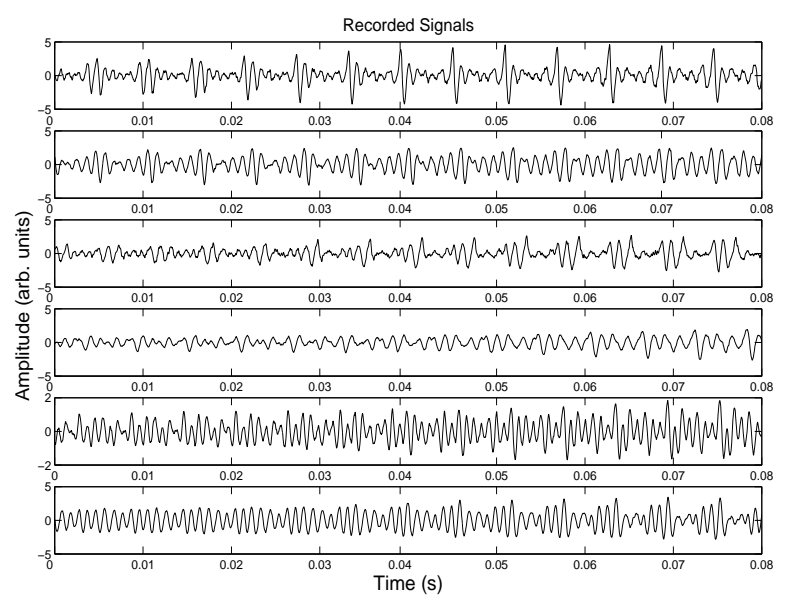

(a)

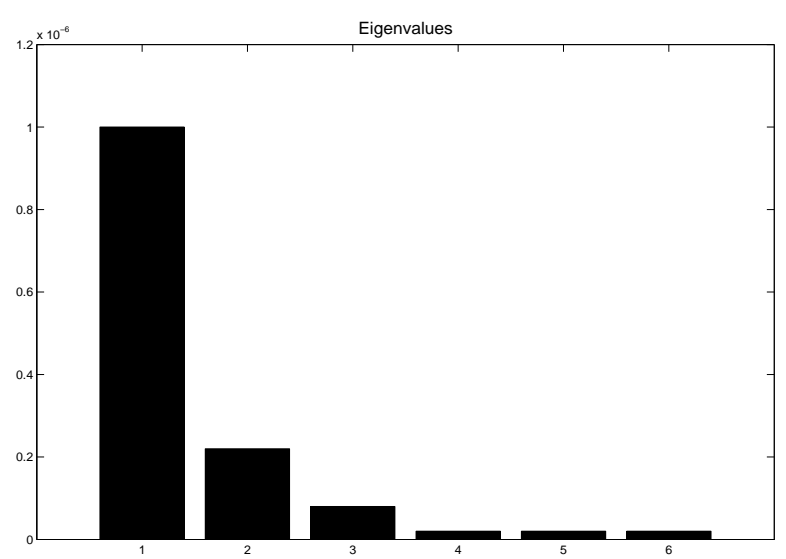

(b)

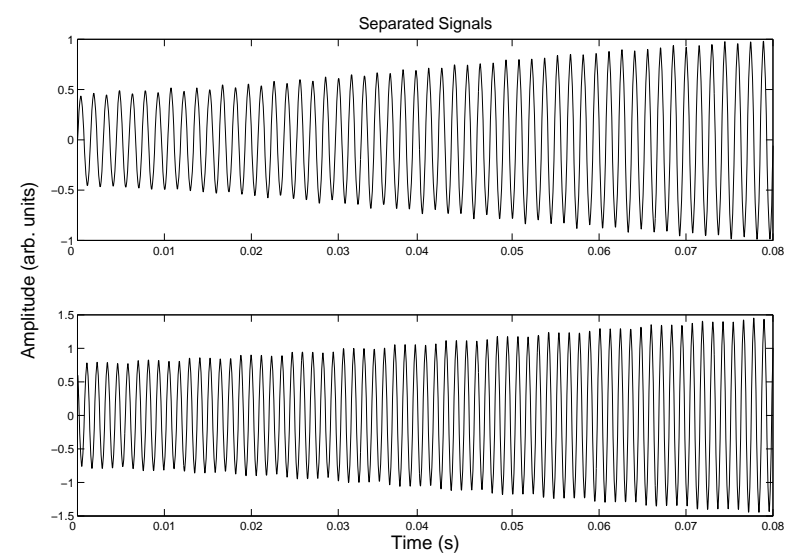

(c)

Figure 5. From top to bottom: Separation related to $\mathrm{E}$ note produced by pipe when two nonlinear modes are enhanced: (a) original signals; (b) PCA: eigenvalues of the covariance matrix; (c) signals separated by ICA with a principal frequency peak at $331 \mathrm{~Hz}$ and a higher peak at $663 \mathrm{~Hz}$.

valve characteristic and $-u_{0}$ is the cut-off voltage. This system can be arranged in order to get the following very

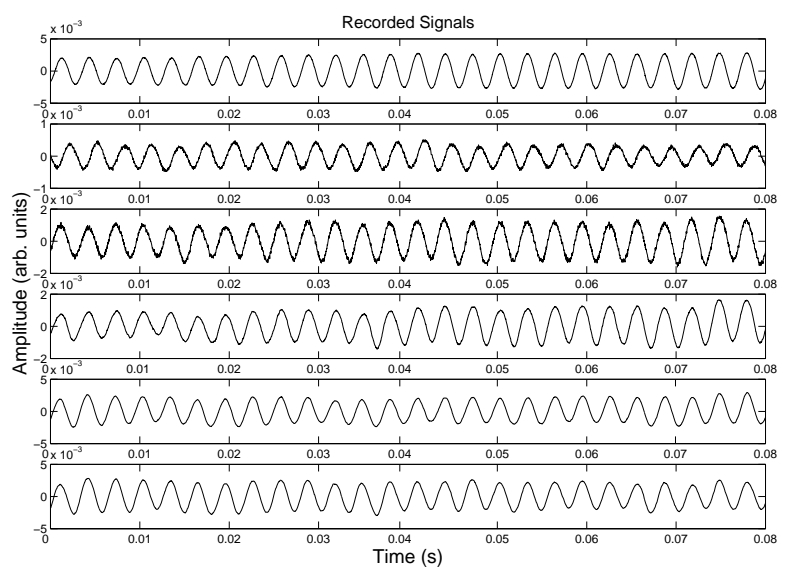

(a)

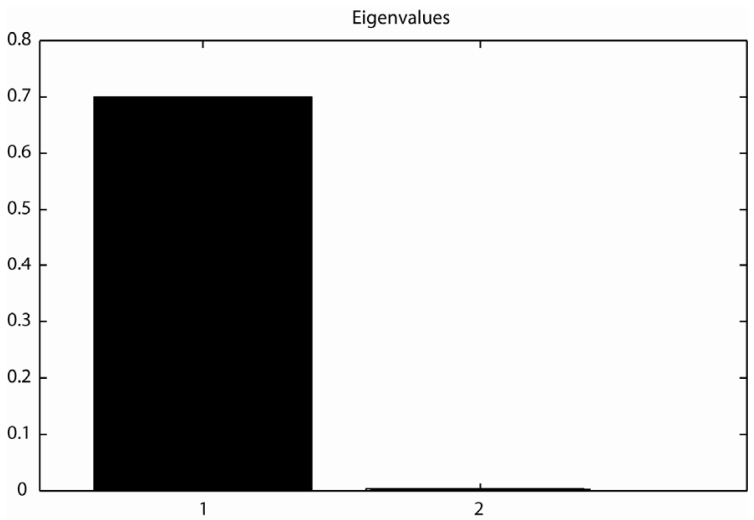

(b)

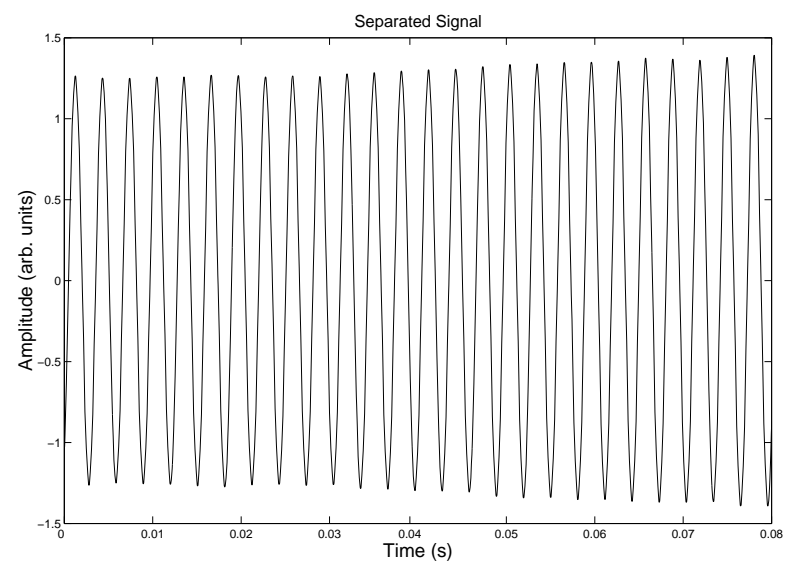

(c)

Figure 6. Separation related to $E$ note produced by pipe when one nonlinear mode is enhanced: (a) original signals; (b) PCA: eigenvalues of the covariance matrix; (c)signal separated by ICA with principal nonlinear mode at $331 \mathrm{~Hz}$.

general Equations:

$$
\begin{array}{ll}
\ddot{x}+2 h_{1} \dot{x}+\omega_{0}^{2} x=0 & \text { for } x<b \\
\ddot{x}+2 h_{2} \dot{x}+\omega_{0}^{2} x=0 & \text { for } x>b
\end{array}
$$


where $b$ is the hopping threshold in which the nonlinearity of the system is concentrated, $\omega_{0}^{2}=1 / L C$ is the natural frequency, $h_{1}=\left(\omega_{0}^{2} / 2\right) R C$ and $h_{2}=\left(\omega_{0}^{2} / 2\right)$ $(M S-R C)$. The latter parameters represent the dissipative and pumping parameters. The phase space is divided by a straight line $x=b$ into different regions identified by the two differential equations of the system [22]. Notice that self-oscillations can be installed for suitable parameters and only if the threshold is negative. Apart from the derivation of this model based on electric circuits, formally the equations can hold for every system, including organ pipe, in which self-oscillations are settled by the competition of a dissipative and a pumping parameter. A discussion of the true physical meaning of $h_{1}, h_{2}$ is out of our purpose, since our modeling is, at the present stage, only analogical. But it is clear that these two parameters represent, in an effective way, pumping due to the blowing and all the typical dissipating effects always present in the organ pipe sound production.

\subsection{Synthesized Sound}

We simulate the three nonlinear modes separately. First step is to filter the recorded $E, C, G$ notes. We fix the best pass-band width by comparing the filtered signals with the ICs. We remark that the filtered signals have high correlation with the signals obtained by the ICA analysis previous explained. To estimate the parameters $h_{1}, h_{2}$ and $\omega$ of the single Andronov oscillator, we construct a 3-dimensional matrix, whose elements generate, separately, a signal that can be compared to the original filtered one.

We choose the best 3-tuple in the sense of minimum square, i.e., we fix those parameters that generate a minimum root mean square deviation with respect to the reference signal. The value of $\omega$ corresponds, within the statistical errors, to the frequency of the excited mode. We report the detailed analysis only for $C$ note. Table 1 contains all the parameters for the other notes. Regarding $C$, the first Andronov oscillator, corresponding to the first nonlinear mode, has the following parameters: $h_{1}=$ $1630, h_{2}=450, b=-0.005$. In this case, the best correlation coefficient between source signal and simulated one is 0.9893 as you can see in Figure 8(a). In Figure 8(b), the phase spaces of recorded and simulated signals confirm such a good similarity between the two signals. In this way we obtained not only the frequency information of the signal but we reproduced exactly the waveform of the sound as we can see from the attack phase. The same analysis has been made for the mode with lower frequency $(263 \mathrm{~Hz})$ and the mode with higher frequency $(1046 \mathrm{~Hz})$. In the two cases, the parameters are respectively $h_{1}=1230, h_{2}=320, \mathrm{~b}=-0.0013$ and $h_{1}=1100, h_{2}$ $=430, b=-0.0055$. The correlation coefficients are 0.86
Table 1. All the parameters obtained on the basis on the best RMS relative to different notes.

\begin{tabular}{ccccccc}
\hline \multirow{2}{*}{ mode } & \multicolumn{3}{c}{$E$} & & \multicolumn{3}{c}{$G$} \\
\cline { 2 - 7 } & $1^{\text {st }}$ & $2^{\text {nd }}$ & $3^{\text {rd }}$ & $1^{\text {st }}$ & $2^{\text {nd }}$ & $3^{\text {rd }}$ \\
\hline$h_{1}$ & 1200 & 250 & 1200 & 1200 & 250 & 1200 \\
$h_{2}$ & 350 & 100 & 500 & 350 & 100 & 500 \\
$b$ & -0.075 & -0.001 & -0.06 & -0.035 & -0.013 & -0.008 \\
$f_{0}(\mathrm{~Hz})$ & 331 & 663 & 165 & 397 & 785 & 198 \\
corr & 0.92 & 0.96 & 0.97 & 0.99 & 0.96 & 0.83 \\
\hline
\end{tabular}

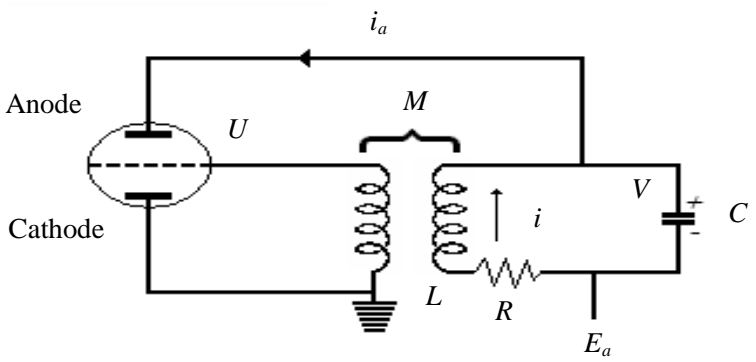

Figure 7. Valve generator with the resonant network in the anode circuit.

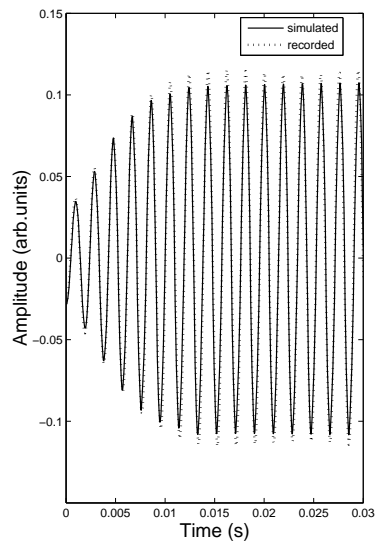

(a)

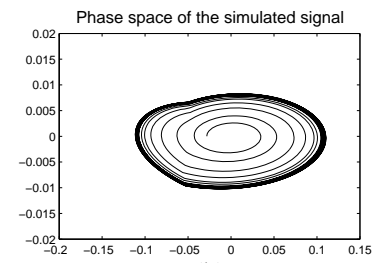

(b)

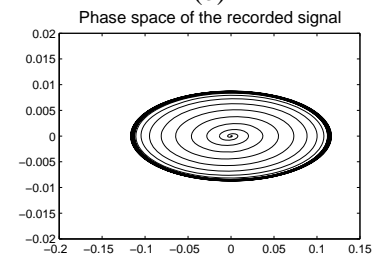

(c)
Figure 8. Phenomenological Dynamical system reproducing the $C$ sound: (a) Comparison between the recorded signal and the filtered one at $523 \mathrm{~Hz}$ (solid line) and the respective synthetic signal (dot); (b) Phase spaces of the synthetic signal; (c) Phase spaces of the recorded signal filtered at 523 Hz.

and 0.98 , respectively. The correlation between simu lated and original signal is very high as in the case of an harmonic oscillator, but the quality of the sound, in the listening, is improved, giving the full tone of the original note w.r.t the metallic sound, due to the coupling of 3 harmonic oscillators. In this way we obtained a nonlinear waveform that is highly correlated to the envelope of a 
pipe sound. The main idea, now, is to use these features to analogical reproduce the real timbre of the pipe sound. By comparing the synthesized waveform with that obtained from a simple additive synthesis we obtain a more realistic waveform and sound.

\subsection{AB-Preference Test}

To validate the introduced nonlinear model we perform a formal AB-preference test [11] versus the linear oscillator based model and the recorded notes. In these tests we select 10 listeners and they are subjected to several notes processed by the two systems in randomized order. For each note the listeners have made a preference decision.

In details, in Table 2 the listeners are invited to make a choice between the modes simulated by using the linear and nonlinear oscillators. Totally, we have 7 notes simulated by the two models. Each note has been simulated by the two models varying the number of modes from 1 to 3 . We see from these results that a nonlinear model is preferred in all the cases with higher order percentages. In Table 3 and Table 4, instead, we have proposed to make a choice between signals obtained by the nonlinear model considering the superposition of one and two or two and three modes, respectively. As confirmed by the ICA analysis we have that the best performance is associated with the modes simulated by using three nonlinear systems linearly superimposed. Finally, in Table 5 we detail the results obtained subjecting the listeners to make a preference between real recorded notes and that

Table 2. AB-preference test between the linear and nonlinear oscillators based models.

\begin{tabular}{cccc}
\hline Mixture & 1 mode & 2 modes & 3 modes \\
\hline Linear & $23 \%$ & $14 \%$ & $7 \%$ \\
Non-Linear & $77 \%$ & $86 \%$ & 93 \\
\hline
\end{tabular}

Table 3. AB-preference test of the nonlinear oscillator varying the number of modes: comparison by using 1 and 2 modes.

\begin{tabular}{cc}
\hline Model & Non-linear \\
\hline 1 mode & $31 \%$ \\
2 modes & $69 \%$ \\
\hline
\end{tabular}

Table 4. AB-preference test of the nonlinear oscillator varying the number of modes: comparison by using 2 and 3 modes.

\begin{tabular}{cc}
\hline Model & Non-Linear \\
\hline 2 modes & $29 \%$ \\
3 modes & $71 \%$ \\
\hline
\end{tabular}

Table 5. AB-preference test between recording notes and that simulated by the non-linear oscillator with three modes.

\begin{tabular}{rc}
\hline & Comparison \\
\hline recorded & $56 \%$ \\
simulated & $44 \%$ \\
\hline
\end{tabular}

simulated by the nonlinear oscillator with three modes. In this case the results show that the proposed model is a good candidate to appropriately reproduce an organ pipe waveform note.

\subsection{Hardware Schematization}

The proposed model permits to obtain a realistic pipe waveform with features very similar to the source pipe (included the attack phase). In other words, it is possible to give an hardware schematization, i.e., design a suitable integrate circuit, observing that the circuit in Figure 7 can be developed in terms of logical functions adopting the scheme of Figure 9 by using the Matlab ${ }^{\mathrm{R}}$ Simulink. From the given scheme we see that the main operations are addition (and substraction) and integration. These operations could be obtained by using an operational amplifier. An operational amplifier is a voltage and current amplifier obtained by using transistors. The circuit based on transistors could be more compact than the valve based one but it is knows that the valve could give a better fidelity.

\section{Conclusions}

In this paper we have analyzed acoustic signals emitted by organ pipes. At a first elementary approximation the generative model of sound in musical instruments seems to be linear. But the analysis and models so produced appear suitable only until the physical processes are investigated in a little more detail. Indeed, the whole system that constitutes a musical instrument is nonlinear.

By using ICA, relevant features of single tones have been extracted from real data (three nonlinear modes). This analysis suggests to use an additive model to synthesize the pipe sounds. The decomposition of the notes and of the chords into independent components provides sufficient information to model the waveform envelope together with the transient attack. This permits to have a better quality sound than that obtained by using simple additive methods based on linear harmonic oscillators or that obtained by using spectral analysis of the sound.

We have introduced a simple and suitable analogical model, able to reproduce the registered waveform and sound in listening. The model provides simulated signals highly correlated with the original one. Furthermore, the quality of the sound, in the listening, is improved, giving 


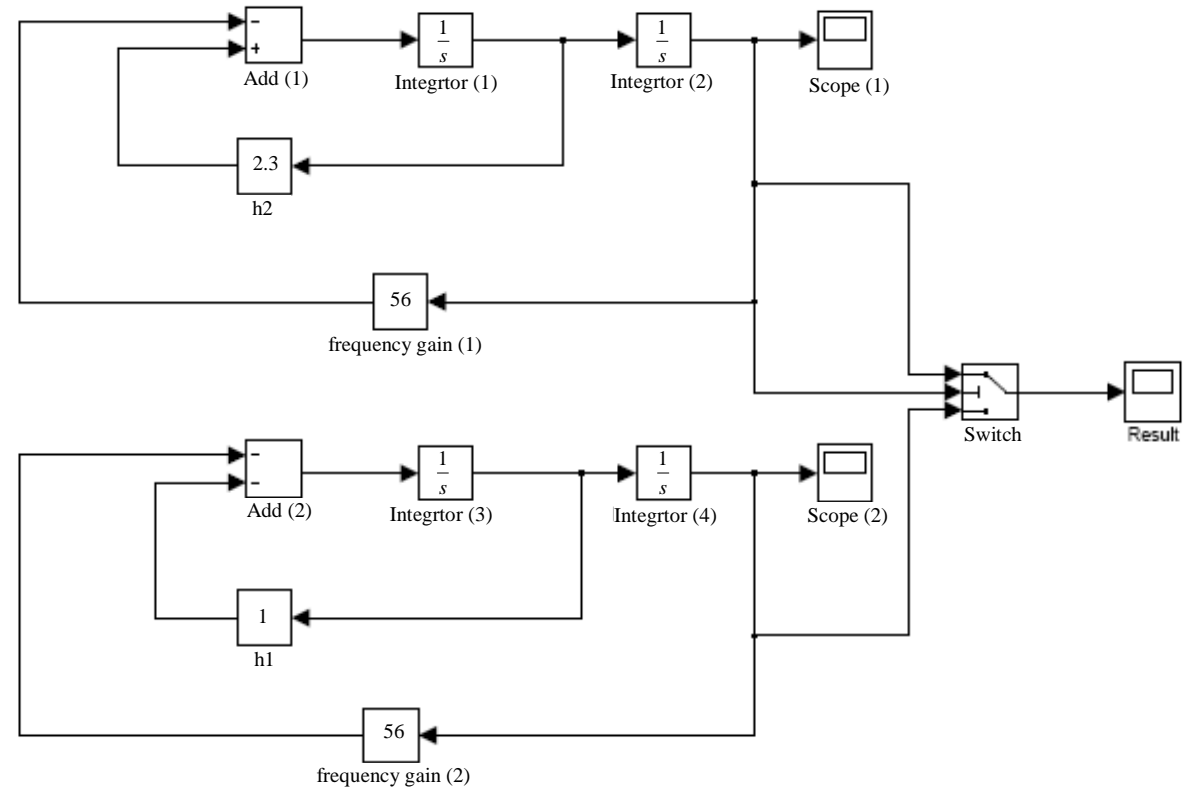

Figure 9. Andronov oscillator obtained by using simulink.

the full tone of the original note compared with the metallic sound, due to the additive synthesis that uses harmonic oscillators. In the next future the authors will focus their attention on the physical realization of the organ pipe system and to apply the analysis and synthesis approach to other musical instruments.

\section{REFERENCES}

[1] P. Gorges, "Programming Synthesizers," Wizoobooks, Bremen, 2005.

[2] N. H. Fletcher and T. D. Rossing, "The Physics of Musical Instruments,” II Edition, Springer, New York, 1998.

[3] N. H. Fletcher, "The Nonlinear Physics of Musical Instruments," Reports on Progress in Physics, Vol. 62, No. 5, 1999, pp. 723-764. doi:10.1088/0034-4885/62/5/202

[4] S. A. Elder, "On the Mechanism of Sound Production in Organ Pipes," Journal of Acoustical Society of America, Vol. 54, No. 6, 1973, pp. 1554-1564. doi:10.1121/1.1914453

[5] A. Hyvarinen, J. Karhunen and E. Oja, "Independent Component Analysis,” Wiley-So Inc., Hoboken, 2001. doi:10.1002/0471221317

[6] S. Osowski, A. Majkowski and A. Cichocki, "Robust PCA Neural Networks for Random Noise Reduction of the Data," IEEE International Conference on Acoustic, Speech, and Signal Processing, Vol. 5, 2000, pp. 3152-3155.

[7] H. D. I. Abarbanel, “Analysis of Observed Chaotic Data," Springer-Verlag, New York, 1995.

[8] E. De Lauro, S. De Martino, E. Del Pezzo, M. Falanga, M. Palo and R. Scarpa, "Model for High-Frequency Strombolian Tremor Inferred by Wavefield Decomposition and Reconstruction of Asymptotic Dynamics," Journal of
Geophysical Research, Vol. 113, 2008, 16 Pages.

[9] F. Takens, "Detecting Strange Attractors in Turbolence," Dynamical Systems and Turbulence Warwick, Lectures Notes in Mathematics, Vol. 898, 1981, pp. 366-381.

[10] A. Ciaramella, E. De Lauro, S. De Martino, M. Falanga and R. Tagliaferri, "ICA Based Identification of Dynamical Systems Generating Synthetic and Real World Time Series,” Soft Computing, Vol. 10, 2006, pp. 587-606. doi:10.1007/s00500-005-0515-7

[11] M. Covell, M. Withgott, and M. Slaney, "Mach1: Nonuniform Timescale Modification of Speech," Proceedings of IEEE International Conference on Acoustic, Speech, Signal Processing (ICASSP), Seattle, 1998, pp. 349-352.

[12] E. De Lauro, S. De Martino, E. Esposito, M. Falanga and E. P. Tomasini, “Analogical Model for Mechanical Vibrations in Flue Organ Pipes Inferred by Independent Component Analysis,” Journal of Acoustical Society of America, Vol. 122, No. 4, 2007, pp. 2413-2424. doi:10.1121/1.2772225

[13] F. Acernese, A. Ciaramella, S. De Martino, R. De Rosa, M. Falanga and R. Tagliaferri, "Neural Networks for Blind Source Separation of Stromboli Explosion Quakes,” IEEE Transactions on Neural Networks, Vol. 14, No. 1, 2003, pp. 167-175. doi:10.1109/TNN.2002.806649

[14] A. Ciaramella, C. Bongardo, H. D. Aller, M. F. Aller, G. De Zotti, A. Lahteenmaki, G. Longo, L. Milano, R. Tagliaferri, H. Terasranta, M. Tornikoski and S. Urpo, "A Multifrequency Analysis of Radio Variability of Blazars,” Astronomy \& Astrophysics Journal, Vol. 419, 2004, pp. 485-500.

[15] A. Ciaramella, "Single Channel Polyphonic Music Transcription," Frontiers in Artificial Intelligence and Applications, New Directions in Neural Networks-18th Italian Workshop on Neural Networks: WIRN 2008, Vol. 193, 2009, pp. 99-108. 
[16] E. De Lauro, S. De Martino, M. Falanga, A. Ciaramella and R. Tagliaferri, "Complexity of Time Series Associated to Dynamical Systems Inferred from Independent Component Analysis,” Physical Review E, Vol. 72, 2005, pp. 1-14.

[17] A. Ciaramella, M. Funaro and R. Tagliaferri, "Separation of Convolved Mixtures in Frequency Domain ICA,” International Journal of Contemporary Mathematical Sciences, Vol. 1, No. 16, 2006, pp. 769-795.

[18] A. Ciaramella and R. Tagliaferri, “Amplitude and Permutation Indeterminacies in Frequency Domain Convolved ICA," Proceedings of the IEEE International Joint Conference on Neural Networks 2003, Vol. 1, 2003, pp. 708713.
[19] B. Natarajan, "Filtering Random Noise from Deterministic Signals via Data Compression," IEEE Transactions on Signal Processing, Vol. 43, 1995, pp. 2595-2605. doi:10.1109/78.482110

[20] P. Grassberger and I. Procaccia, "Measuring the Strangeness of Strange Attractors," Physica D: Nonlinear Phenomena, Vol. 9, No. 1-2, 1983, pp. 189-208.

[21] L. D. Landau and E. M. Lifshitz, "Fluid Mechanics,” Pergamon Press, Oxford, 1959.

[22] A. A. Andronov, A. A. Vitt and S. E. Khaikin, "Theory of Oscillators,” Republished Dover Publications, Inc., 1966. 